\title{
Erythema ab igne
}

\author{
Luca Naldi • Andrea Berni • Nicola Pimpinelli • \\ Loredana Poggesi
}

Received: 11 March 2010/Accepted: 7 April 2010/Published online: 8 May 2010

(C) SIMI 2010

\section{Case report}

An 86-year-old woman was referred to our Clinic because of a new onset of anaemia. She was under treatment with warfarin for chronic atrial fibrillation. The INR at admission was 4.1. Her caregiver excluded hematemesis or melena, and assured no NSAID had been taken. She appeared pale and was haemodynamically stable. A wellmarked, reddish-brown, netlike discoloration of the skin, with clear-cut margins was present on the anterior and medial surfaces of both thighs (Fig. 1). This kind of lesion was not present in any other body areas. Physical examination was otherwise normal.

Routine blood examination revealed the presence of a microcytic anaemia (haemoglobin $7.8 \mathrm{~g} / \mathrm{dL}$; mean corpuscular volume $60.8 \mathrm{fL}$ ) with iron deficiency (serum iron $20 \mu \mathrm{g} /$ $\mathrm{dL}$; serum ferritin $30 \mu \mathrm{g} / \mathrm{L}$ ). The erythrocyte sedimentation rate appeared high $(75 \mathrm{~mm} / \mathrm{h})$. Lupus anticoagulant panel, antinuclear antibodies, Coombs tests, cryoglobulins and antiHCV antibodies were normal. Haptoglobin levels were normal. Esophagogastroduodenoscopy showed a sliding hiatal hernia with erosive esophagitis.

The patient reported that the skin discoloration had appeared on her thighs many years before, and was chronic and stable. She added she would usually keep a hot water bottle on her thighs. It is to be noted that the extension of the lesion corresponded to the surface of the water bottle.

Therefore, the skin discoloration and anaemia likely had no relationship. In fact, the anaemia was secondary to

L. Naldi · A. Berni $(\bowtie) \cdot$ N. Pimpinelli $\cdot$ L. Poggesi Department of Medical and Surgical Critical Care, AOU Careggi, University of Florence, Viale G.B. Morgagni, 85, 50134 Florence, Italy e-mail: berniandrea@inwind.it

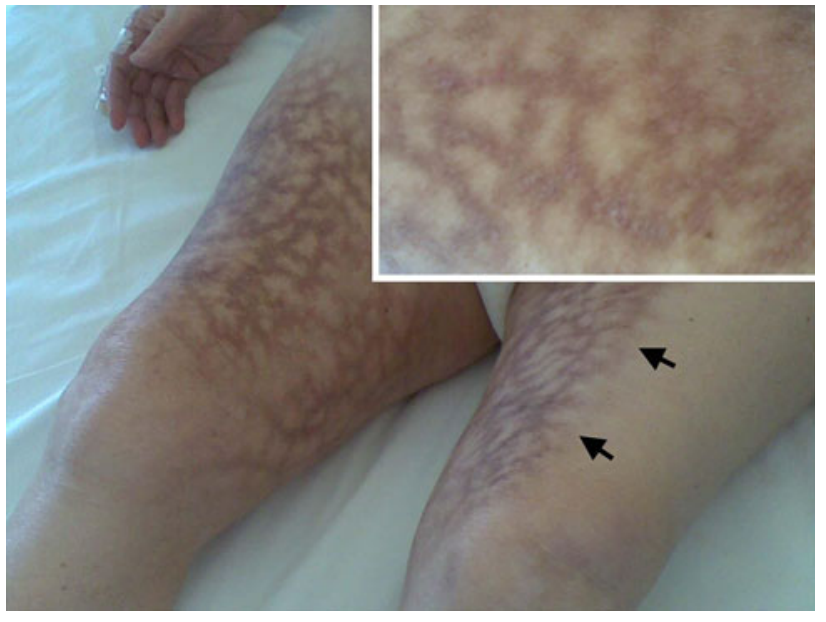

Fig. 1 A well-marked, reddish-brown, netlike discoloration of the skin, with clear-cut margins (black arrows) is present on the anterior and medial surfaces of patient's thighs. A magnification of the skin lesion is shown in the box at the top right corner

erosive esophagitis, and was treated with iron supplementation and proton pump inhibitors.

\section{Discussion}

Erythema ab igne appears as a reticulated, erythematous, hyperpigmented eruption that may occur after prolonged and repeated skin exposure to mild heat or infrared radiation, but under the threshold of thermal burn [1]. It can be also characterized by skin atrophy, telangiectasia and subepidermal bullae, and it is usually asymptomatic. Distribution and contour of the skin lesions depend on the direction of the incident radiation, and the interposition of clothing. 
Erythema ab igne has been a common condition seen on the legs of people who have been standing or sitting close to a fire. Since the availability of central heating system in most buildings has become widespread, in modern societies its frequency has decreased. Nowadays it is occasionally still seen on localized areas of the body repeatedly exposed to the different heat sources currently in use. It can develop on the shins and inner thighs of subjects who sit close to heaters or stoves, or using hot bottles for a long time, but also after prolonged and close contact with a car heater. Recently, erythema ab igne has been reported to appear in patients working for several hours with a laptop computer placed on their thighs. It also develops on the lumbar region and abdomen in patients using a heat source, such as heating pad to treat chronic back and abdominal pains, or for aesthetic reasons against cellulite. When it appears on the face and arms of cookers and bakers, it may be considered as an occupational hazard.

The differential diagnosis, which includes livedo reticularis, port-wine stain and poikiloderma, does not usually require skin biopsy. In fact, the patients' history of chronic heat exposure, and the distribution and distinctive appearance of cutaneous changes are pathognomonic. A biopsy may be taken if any evidence of cutaneous malignancy appears, such as nodules or ulceration. Indeed, squamous cell carcinoma and Merkel cell carcinoma can develop in areas of erythema ab igne although rarely [2].

The mainstay of the treatment of erythema ab igne is to remove the source of infrared radiation. However, if exposure to the infrared radiation is not discontinued, pigmentary abnormalities may persist and cutaneous atrophy or malignancy may ensue.

Undoubtedly, macroscopic skin characteristics of erythema ab igne are similar to those of livedo reticularis, which appears as a netlike, violaceous discoloration of the skin, typically on the legs [3].

Livedo reticularis can be secondary to numerous and heterogeneous causes and systemic diseases [4]. Differential diagnosis should especially focus on elements that suggest potential autoimmune conditions or thrombotic disorders. In fact, livedo reticularis can be associated with autoimmune (in particular lupus erythematosus), thrombotic or vessel wall diseases [4], but has not been reported to be related to heat exposure [4].

Livedo reticularis is caused by increased prominence of the venous beds in the skin, either from impediments to arterial inflow, venous dilatation, or obstruction to venous outflow [3]. General histopathologic features in skin biopsies mainly show red cell sludging and vessel wall inflammation with arterial obliteration, with or without thrombi, vessel wall thickening and fibrinoid changes [5].

The histopathologic characteristics of erythema ab igne are nonspecific and include epidermal atrophy, hyperkeratosis, abundant melanophages in dermis, elastic fibres alterations and perivascular infiltration of inflammatory cells, mainly composed of lymphocytes, and dilatation of dermal blood vessels [1].

To date, the pathophysiology of erythema ab igne is not well known. Some patients may represent a predisposition to the appearance of erythema ab igne, possibly on the basis of an immunological background. A local "vasculitis ab igne" of the skin therefore may be hypothesized, in terms of a latent vascular autoimmunity triggered by a chronic exposure to the heat noxa.

\section{Conflict of interest None.}

\section{References}

1. Cavallari V, Cicciarello R, Torre V, Gagliardi ME, Albiero F, Palazzo R, Siragusa M, Schipis C (2001) Chronic heat-induced skin lesions (erythema ab igne): ultrastructural studies. Ultrastruct Pathol 25:93-97

2. Hewitt JB, Sherif A, Kerr KM, Stankler L (1993) Merkel cell and squamous cell carcinomas arising in erythema ab igne. $\mathrm{Br} \mathrm{J}$ Dermatol 128:591-592

3. Kroshinsky D, Stone JH, Bloch DB, Sepehr A (2009) Case records of the Massachusetts General Hospital. Case 5-2009. A 47-yearold woman with a rash and numbness and pain in the legs. N Engl J Med 360:711-720

4. Gibbs MB, English JC 3rd, Zirwas MJ (2005) Livedo reticularis: an update. J Am Acad Dermatol. 52:1009-1019

5. In SI, Han JH, Kang HY, Lee ES, Kim YC (2009) The histopathological characteristics of livedo reticularis. J Cutan Pathol 36:1275-1278 